\title{
Original
}

\section{Facial Aesthetic Analysis of Prosthetic Recovery after Partial Maxillectomy Using a Non-Contact 3-Dimensional Digitiser}

\author{
Tomoyuki Kanbe ${ }^{1}$, Satoshi Yokoo ${ }^{1}$, Masaru Ogawa ${ }^{1}$, Yu Takayama ${ }^{1}$, Akinori Gomi ${ }^{1}$ and Takaya \\ Makiguchi $^{1}$ \\ 1 Department of Oral and Maxillofacial Surgery - Plastic Surgery, Gunma University Graduate School of Medicine, 3-39-22 Showa-machi, \\ Maebashi, Gunma 371-8511, Japan
}

\begin{abstract}
Background \& Aims: The purpose of this study was to investigate changes in facial morphology after partial maxillectomy and recovery after dento-maxillary prosthesis placement. Methods: For facial morphology measurements, the non-contact 3-dimensional (3D) digitiser and the analytical software were used for 21 patients with defects classified into Class I or II of the Aramany classification. Results: It was quantitatively demonstrated that facial deformities resulting from partial maxillectomy were due to concavities from the subnasal point to the mentolabial groove in the vertical range, and from the region around the nasal alar to the anterior cheek in the lateral range. Facial features anterolaterally expanded on the defected side in volume with the prosthesis. Facial disfigurement extended to the lower face, and improvement of these deformities was also observed with the prosthesis. Using the non-contact 3D digitiser, the direction and amount of movement of the facial components within the mid- and the lower face regions were 3-dimensionally determined, enabling rapid and accurate identification of facial deformities, and recovery of the facial morphology with prosthesis. Conclusion: This is the first study to analyse of prosthesis-induced changes in facial morphology after partial maxillectomy with a non-contact 3D digitiser.
\end{abstract}

\begin{tabular}{|c|}
\hline $\begin{array}{l}\text { Key words: } \\
\text { dento-maxillary prosthesis, } \\
\text { partial maxillectomy, } \\
\text { non-contact 3-dimensional digitiser, } \\
\text { facial morphology, } \\
\text { principal component analysis }\end{array}$ \\
\hline $\begin{array}{cl}\text { Publication } & \text { history: } \\
\text { Received: } & \text { August 21, 2017 } \\
\text { Revised: } & \text { September 29, } 2017 \\
\text { Accepted: } & \text { October 3, 2017 }\end{array}$ \\
\hline $\begin{array}{l}\text { Corresponding author: } \\
\text { Satoshi Yokoo } \\
\text { Department of Oral and Maxillofacial Surgery • Plastic } \\
\text { Surgery, Gunma University Graduate School of Medicine, } \\
\text { 3-39-22 Showa-machi, Maebashi, Gunma 371-8511, Japan } \\
\text { Tel: +81-27-220-8484 } \\
\text { E-mail: syokoo@ gunma-u.ac.jp }\end{array}$ \\
\hline
\end{tabular}

\begin{abstract}
Introduction
Midface deformities after maxillectomy markedly influence patient's social lives. ${ }^{1}$ Facial morphology and function are recovered by combining reconstructive surgery ${ }^{2-4}$ and/or dento-maxillary prosthesis ${ }^{5,6}$ after maxillectomy. The dento-maxillary prosthesis may be simply adjusted and thus accommodates facial changes over time ${ }^{1,7,8}$; however, there are no objective evaluations of the aesthetic effects of these prostheses. The aesthetic goal of the dento-maxillary prosthesis is based on the aesthetic judgments of patients and physicians, and in many cases, these goals differ. Therefore, a simple and objective method for evaluating facial morphology is necessary for creating a common viewpoint for the patient and treating physician. ${ }^{9}$

The purpose of this study was to investigate changes in facial morphology after partial maxillectomy and recovery after dento-maxillary prosthesis placement. We also sought to establish an objective method for evaluating prosthesis placement effectiveness from an aesthetic viewpoint as a pilot study.

The non-contact 3-dimensional (3D) digitiser is a new precise analytical apparatus used in the fields of oral and maxillofacial surgery, plastic and reconstructive surgery, aesthetic surgery and dentistry. ${ }^{10-14}$ This is the first report on facial morphology changes resulting from dento-alveolar prosthesis placement after partial maxillectomy analysed with a non-contact 3D digitiser.
\end{abstract}


Table 1 Patients

\begin{tabular}{|c|c|c|c|c|c|c|c|}
\hline Case & Age & Sex & Diagnosis & $\begin{array}{l}\text { maxillary } \\
\text { resection }\end{array}$ & $\begin{array}{c}\text { Aramany } \\
\text { classification }\end{array}$ & $\begin{array}{c}\text { Defect of } \\
\text { buccal mucosa }\end{array}$ & $\begin{array}{l}\text { Reconstruction } \\
\text { of buccal mucosa }\end{array}$ \\
\hline 1 & 82 & F & SCC & Partial & I & + & D-P $※$ flap \\
\hline 2 & 69 & M & SCC & Partial & II & - & Skin graft \\
\hline 3 & 68 & $\mathrm{~F}$ & $\mathrm{SCC}$ & Partial & II & - & Reefing \\
\hline 4 & 81 & M & $\mathrm{SCC}$ & Partial & I & + & Skin graft \\
\hline 5 & 67 & M & Malignant melanoma & Partial & II & - & Forearm flap \\
\hline 6 & 65 & M & Malignant melanoma & Partial & II & - & Reefing \\
\hline 7 & 68 & $\mathrm{~F}$ & SCC & Partial & I & + & Forearm \\
\hline 8 & 83 & M & SCC & Partial & I & + & Skin graft \\
\hline 9 & 81 & M & SCC & Partial & $\mathrm{I}$ & + & Skin graft \\
\hline 10 & 63 & M & $\mathrm{SCC}$ & Partial & I & + & Reefing \\
\hline 11 & 77 & M & SCC & Partial & II & - & Reefing \\
\hline 12 & 41 & M & Odontogenic Myxoma & Partial & II & - & Skin graft \\
\hline 13 & 86 & $\mathrm{~F}$ & SCC & Partial & II & - & Reefing \\
\hline 14 & 80 & $\mathrm{~F}$ & SCC & Partial & $\mathrm{I}$ & + & Skin graft \\
\hline 15 & 91 & M & SCC & Partial & I & + & Skin graft \\
\hline 16 & 61 & M & SCC & Partial & II & - & Reefing \\
\hline 17 & 70 & M & SCC & Partial & II & - & Skin graft \\
\hline 18 & 75 & $\mathrm{~F}$ & $\mathrm{SCC}$ & Partial & II & - & Reefing \\
\hline 19 & 66 & M & SCC & Partial & I & + & Reefing \\
\hline 20 & 62 & $\mathrm{~F}$ & SCC & Partial & I & + & Skin graft \\
\hline 21 & 74 & $\mathrm{~F}$ & $\mathrm{SCC}$ & Partial & II & - & Skin graft \\
\hline
\end{tabular}

※ SCC: Squamous cell carcinoma, D-P: Deltopectoralis
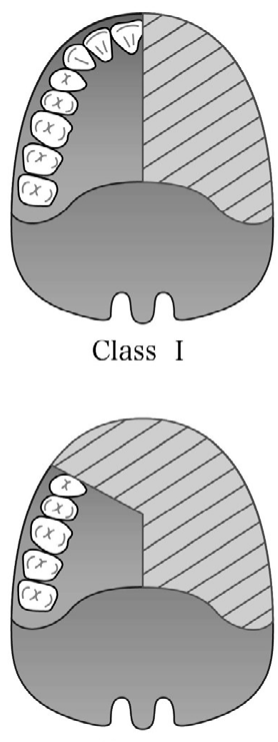

Class IV

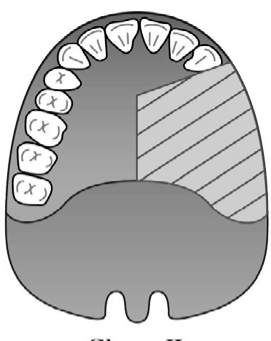

Class II

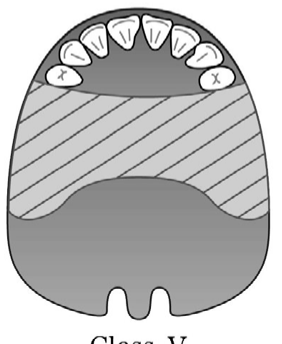

Class V

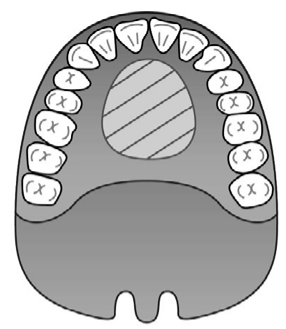

Class III

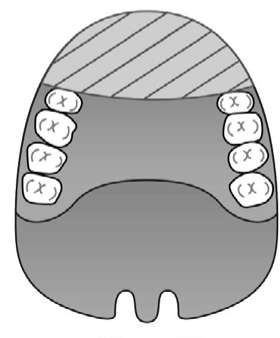

Class VI

Fig. 1 Aramany classification

The Class I and II patients of Aramany classification were analysed. The buccal mucosa was concomitantly resected and reconstructed by skin flap or skin grafting in all Class I patients

\section{Materials and Methods}

The Gunma University Ethics Committee approved all aspects of this study (No.2017-118).

Fifty-eight patients underwent partial maxillectomy followed by dento-maxillary prosthesis treatment at Department of Oral and Maxillofacial Surgery Gunma University Hospital between 2013 and 2016 were included in this study. There were 21 patients with defects classified into Class I or II of the Aramany classification $^{15,16}$ (Table 1 and Fig. 1), comprising 13 men and 8 women, ranging in age from 41 to 91 years (median: 70 years old). The primary disease was squamous cell carcinoma in 18 patients, malignant melanoma in 2, and odontogenic myxoma in one. The Aramany classification was class I and II in 10 and 11 patients. The buccal mucosa was concomitantly resected and reconstructed by skin flap or skin grafting in all Class I patients.

This study excluded Class III to IV of the Aramany classification for comparison of the laterality of the defective side and the normal side. 

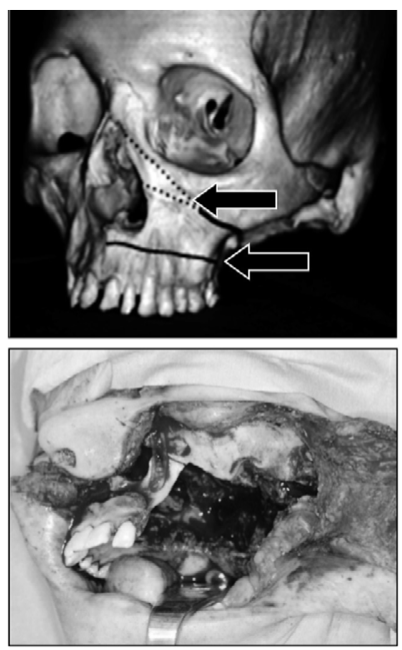

(A)
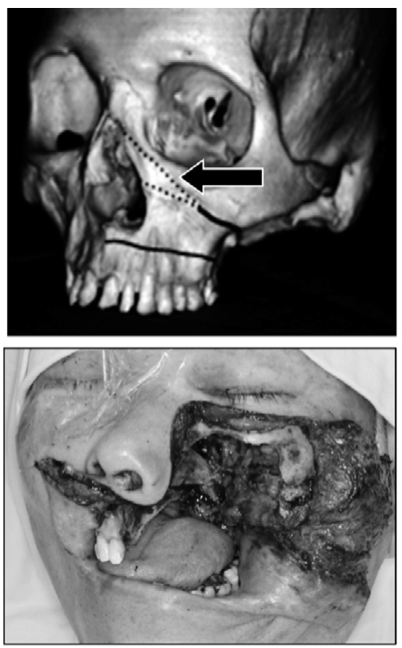

(B)

Fig. 2 Operative procedure of partial maxillectomy and subtotal maxillectomy

We defined resection of the maxillary region lower than the inferior orbital foramen as partial maxillectomy (A), and resection including the inferior orbital foramen conserving the orbital floor as subtotal maxillectomy (B).

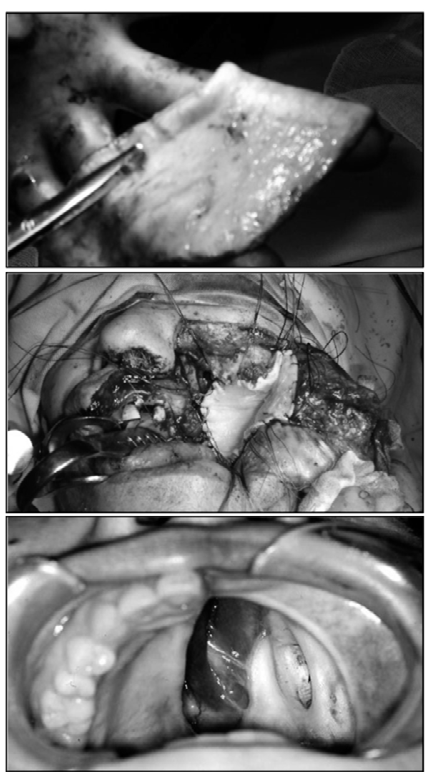

(A)
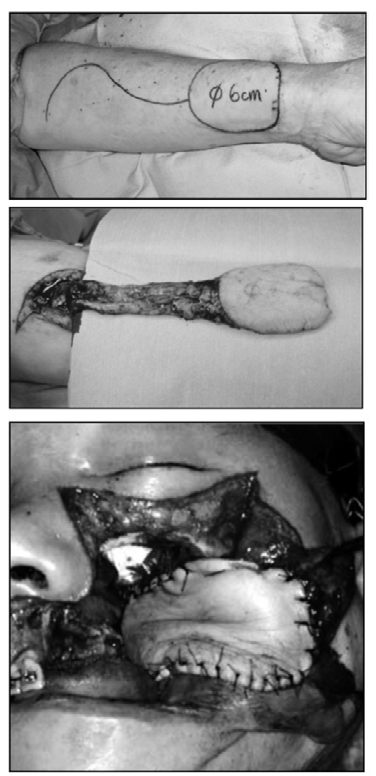

(B)

Fig. 3 Reconstruction of defect of the buccal mucosa for safty margin of the tumour resection

When the safety margin of the resection extends to the buccal mucosa, skin flap transplantation (A: vascularised free forearm flap) or grafting (B) is completed to prevent postoperative scar contractures that result in facial disfigurement.

\section{Operative and prosthetic method for partial maxil- lectomy}

We defined resection of the maxillary region lower than the inferior orbital foramen as partial maxillectomy, and resection including the inferior orbital foramen conserving the orbital floor as subtotal maxillectomy ${ }^{1}$ (Fig. 2). When the safety margin of the resection extends to the buccal mucosa, skin flap trans-

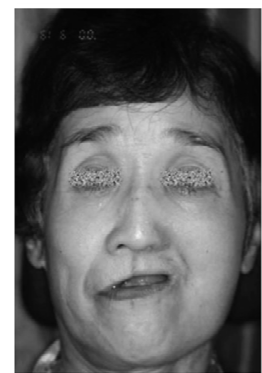

(A)

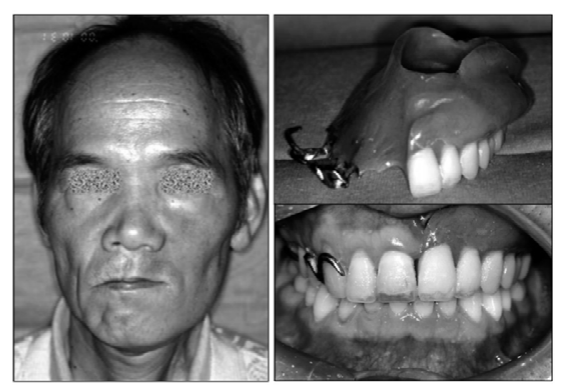

(B)
Fig. 4 Prosthetic procedure for partial maxillectomy For aesthetic change of facial morphology after maxillectomy, reliable 3D identification of defect-induced facial disfigurement and recovery by reconstructive surgery and/ or dento-maxillary prosthesis is important. (A): without prosthesis, (B) with prosthesis.

plantation or grafting is completed to prevent postoperative scar contractures that result in facial disfigurement (Fig. 3). Postoperative maxillary defects are not closed by reconstructive materials (i.e. musculocutaneous flaps ${ }^{2,3,17}$ ). In our institution, as a rule, the defects are closed by the dento-maxillary prosthesis in order to discover tumour recurrence and to deal with changes in the postoperative facial appearance immediately ${ }^{5,6,17,18}$ (Fig. 4).

\section{Reference points, lines, and planes}

For facial morphology measurements, the noncontact 3D digitiser Morpheus 3D (Morpeus, Seongnam, Korea) was used. This device scans surfaces with a semiconductor laser slit beam and acquires images in the frontal and bilateral $45^{\circ}$ directions using a single CCD camera. These image data in the 3 directions are then reconstructed into a $3 \mathrm{D}$ image. The resolution was $0.2 \mathrm{~mm}$, and the acquisition time was eight seconds in each direction. The patient sat on a chair for measurements with the upper half of the body positioned vertically relative to the floor. The patient' $s$ head was fixed on a head rest at a position that minimised the blind area of the slit beam, and two image patterns (with and without prosthesis) were recorded (Fig. 5).

In the analysis, reference points were set based on

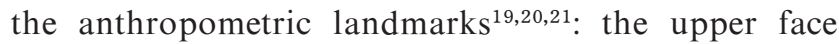
(around the eyelid), entcanthion (En) and ectocanthion (E); midface (around the nasal alar), alare (Al), and subnasale (Sn); lower face (around the lips), stomion (Sto), labrale superius (Ls), labrale inferius (Li), and chelion $(\mathrm{Ch})$. The face was divided into the defective side (D-side) and the normal side (N-side) (Fig. 6).

Then, the coordinate axes and reference planes were established. A straight line passing through the bilateral En was set as the $\mathrm{X}$-axis, and the midpoint between the bilateral En was defined as the coordinate origin point, $\mathrm{O}$. The plane established by the $\mathrm{X}$-axis and $\mathrm{Al}$ on the $\mathrm{N}$-side was regarded as the facial frontal plane. A straight line passing through $\mathrm{O}$ on the facial 


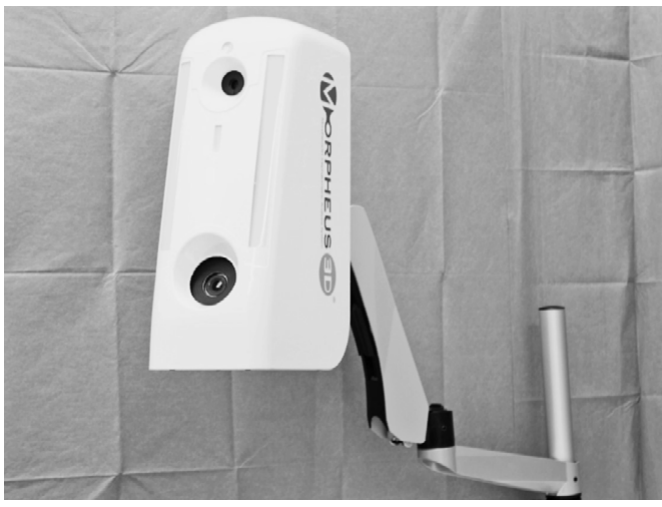

(A)

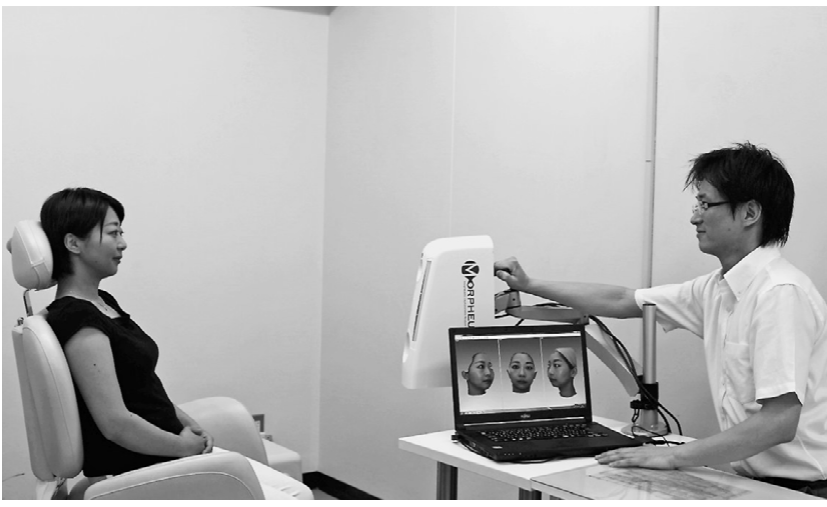

(B)

Fig. 5 Measurement method using the non-contact 3-D digitiser, Morpheus 3-D

Morpheus 3-D scanner (A) scans surfaces with a semiconductor laser slit beam and acquires images in the frontal and bilateral $45^{\circ}$ directions using a single CCD camera. The patient sat on a chair for measurements with the upper half of the body positioned vertically relative to the floor. The patient's head was fixed on a head rest at a position that minimised the blind area of the slit beam, and two image patterns (with and without prosthesis) were recorded (B).

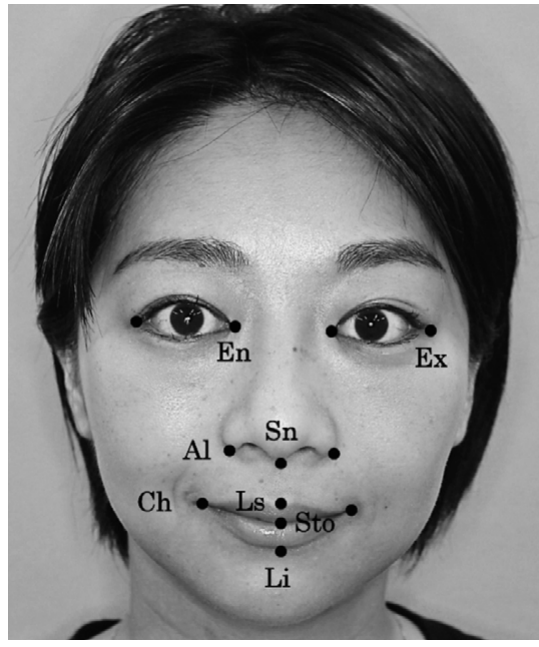

(A)

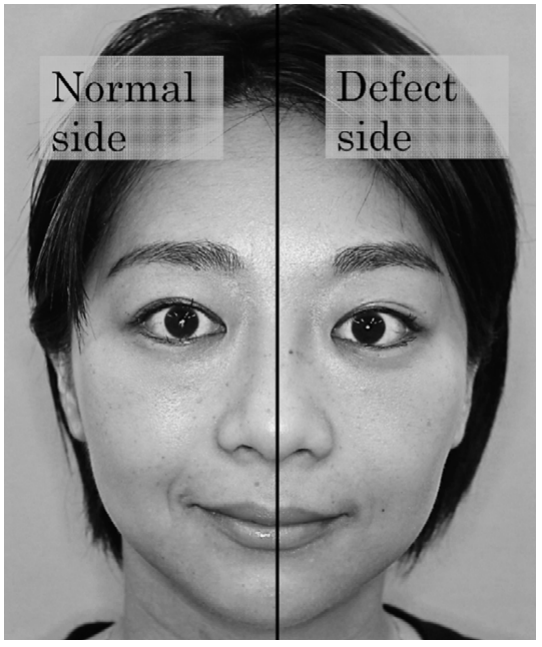

(B)

Fig. 6 Anthropological facial landmarks

Reference points, the upper face (around the eyelid), entcanthion (En) and ectocanthion (Ex); midface (around the nasal alar), alare (Al), and subnasale (Sn); lower face (around the lips), stomion (Sto), labrale superius (Ls), labrale inferious ( $\mathrm{Li})$, and chelion $(\mathrm{Ch})$, were set based on the anthropometric landmarks (A). The face was divided into the defective side (D-side) and the normal side (N-side) (B).

frontal plane vertically to the $\mathrm{X}$-axis was defined as the $\mathrm{Y}$-axis, and a straight line passing through $\mathrm{O}$ positioned vertically to the facial frontal plane was defined as the Z-axis. Only the $\mathrm{X}$-axis was corrected to become a positive vector from the N- to D-side (Fig. 7).

\section{Analysis of facial morphology}

For analysis of facial morphology, the analytical software 3D-Rugle 7 (Medic Engineering Corp, Kyoto, Japan) was used.

A mirror image of the $\mathrm{N}$-side was prepared and matched to the D-side, and the following analyses were carried out (Fig. 8). First, to investigate how the D-side will be changed by prosthesis placement, the

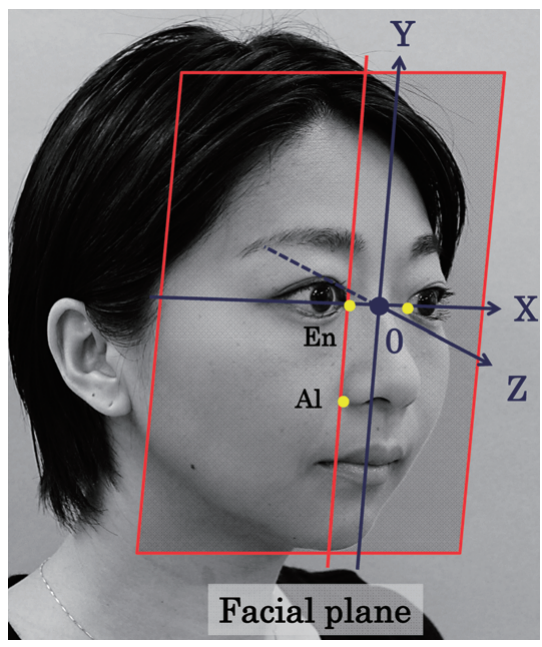

Fig. 7 Reference lines and planes

A straight line passing through the bilateral En was set as the $\mathrm{X}$-axis, and the midpoint between the bilateral En was defined as the coordinate origin point, $\mathrm{O}$. The plane established by the $\mathrm{X}$-axis and $\mathrm{Al}$ on the $\mathrm{N}$-side was regarded as the facial frontal plane. A straight line passing through $\mathrm{O}$ on the facial frontal plane vertically to the $\mathrm{X}$-axis was defined as the $\mathrm{Y}$-axis, and a straight line passing through $\mathrm{O}$ positioned vertically to the facial frontal plane was defined as the Z-axis.

D-side data without prosthesis were superimposed on the D-side data with prosthesis. The differences in the coordinate values $(x, y, z)$ of the reference points on the upper face, midface, and lower face regions were determined, and the direction and amount of movement was analysed. The amount of movement was significant in nine items, and these items were subjected to principal component analysis. ${ }^{22,23}$ The MannWhitney U test was used to compare differences between 2 examination items. A significant difference betwwen the groups if 

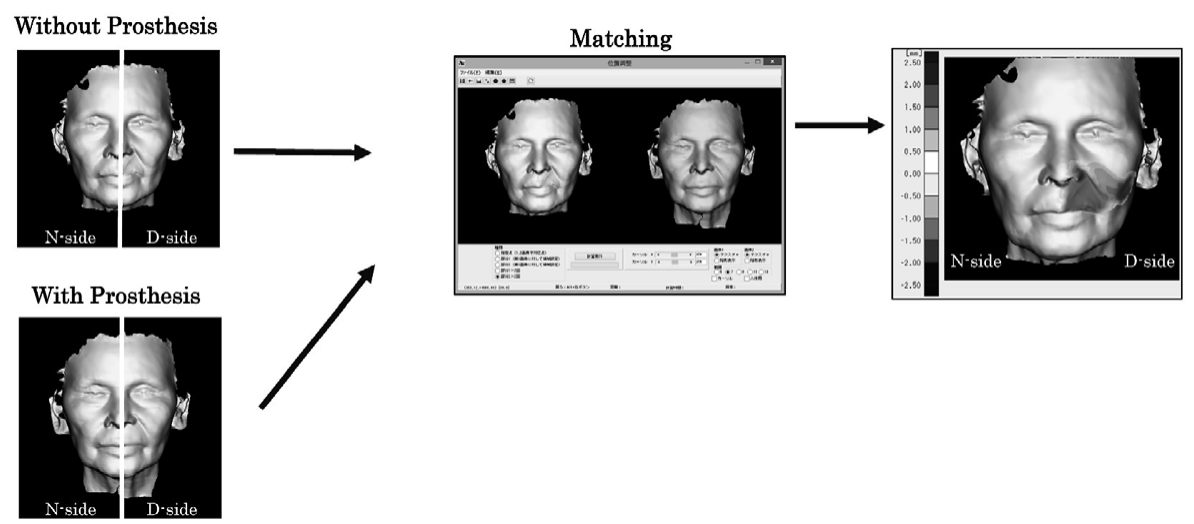

Fig. 8 Facial morphology analytical method

For analysis of facial morphology, the analytical software 3D-Rugle 7 (Medic Engineering Corp, Kyoto, Japan) was used. A mirror image of the N-side was prepared and matched to the D-side, and some analyses were carried out.

Table 2 Change in anthropological facial profile

\begin{tabular}{lrrc}
\hline Point & \multicolumn{1}{c}{$\mathrm{X}$} & $\mathrm{Y}$ & $\mathrm{Z}$ \\
\hline En-D & $0.11 \pm 0.78$ & $-0.07 \pm 0.96$ & $0.19 \pm 0.57$ \\
En-I & $0.09 \pm 0.69$ & $-0.04 \pm 0.73$ & $0.05 \pm 0.43$ \\
Ex-D & $-0.12 \pm 0.86$ & $-0.14 \pm 0.89$ & $0.15 \pm 1.02$ \\
Ex-I & $-0.08 \pm 0.79$ & $0.17 \pm 0.55$ & $0.03 \pm 0.76$ \\
Sn & $0.29 \pm 0.81$ & $0.21 \pm 1.49$ & $2.13 \pm 2.48^{*}$ \\
Al-D & $-0.47 \pm 2.02$ & $0.59 \pm 1.01$ & $2.85 \pm 2.26^{* *}$ \\
Al-I & $0.12 \pm 1.17$ & $0.17 \pm 1.41$ & $0.94 \pm 1.31$ \\
Sto & $0.55 \pm 1.01^{*}$ & $1.59 \pm 2.32^{*}$ & $0.66 \pm 2.71$ \\
Ls & $0.39 \pm 1.06$ & $1.37 \pm 1.22^{* *}$ & $2.98 \pm 3.89^{* *}$ \\
Li & $0.33 \pm 1.31$ & $1.46 \pm 1.05^{* *}$ & $0.37 \pm 2.84$ \\
Ch-D & $1.16 \pm 1.94$ & $2.37 \pm 2.19^{* *}$ & $1.04 \pm 2.24$ \\
Ch-I & $-0.07 \pm 2.38$ & $0.39 \pm 3.76$ & $0.81 \pm 2.47$ \\
\hline \multicolumn{2}{c}{ mean \pm standard deviation } \\
& & \multicolumn{2}{c}{ Significant difference ${ }^{*} \mathrm{p}<0.05 *$ p $<0.01$}
\end{tabular}

the test showed a p-value less than 0.05. In addition, using the statistical analysis software, Statflex ver. 6 (Artech. Co., Ltd., Osaka, Japan), the solutions of characteristic equations were determined using the variance-covariance matrix prepared by standardizing the variables. The eigenvalue, contribution ratio, accumulated contribution ratio, and factor loading were output. The principal component score was calculated from the product of the standardised variables and factor loading.

Next, the recovery of facial morphology was investigated as follows: 1) the distance and the volume of the gap between superimposed facial surface generated on the D-side were analysed as prosthesis placementinduced changes; 2) the N-side data with and without prosthesis were superimposed to investigate the influence of prosthesis, and 3) the D-side data with prosthesis were matched to $\mathrm{N}$-side data to investigate recovery of facial asymmetry resulting from prosthesis placement.

\section{Results}

\section{Direction and amount of facial component move- ment by dento-maxillary prosthesis}

In the upper face, no significant changes were noted at the entcanthion (En) or ectocanthion (Ex) on either the D- or N-sides. At midface, prosthesis placement significantly moved the subnasale $(\mathrm{Sn})$ and alare points on the D-side (Al-D) toward the positive side in the direction of $\mathrm{Z}$-axis. In the lower face, the stomion (Sto) and the labrale superius (Ls) significantly moved toward the positive side in the directions of $\mathrm{X}$ - and Y-axis. The chelion, the oral commissure, on the D-side (Ch-D), significantly moved toward the positive side in the direction of $\mathrm{Y}$-axis (Table 2).

The amount of movement was significant for nine items, all of which were subjected to principal component analysis. The eigenvalue was 1.0 or greater up to the 4 th principal component, and the accumulated contribution ratio was $83 \%$. Thus, the factor loading of the 1 st to 4 th principal components was determined. The absolute values of factor loadings higher than 0.4 are shown in Table 3. In the 1st principal component, the factor loading of two variables was high: positive 
Table 3 Principal component analysis

\begin{tabular}{|c|c|c|c|c|}
\hline Point & Comp. 1 & Comp. 2 & Comp. 3 & Comp. 4 \\
\hline $\operatorname{Sn}(Z)$ & 0.2500 & 0.0383 & 0.6488 & 0.3249 \\
\hline AlD (Z) & 0.2326 & -0.1673 & -0.3238 & 0.6726 \\
\hline Sto $(\mathrm{X})$ & -0.0115 & 0.4989 & -0.1873 & 0.4664 \\
\hline Sto $(\mathrm{Y})$ & -0.2391 & 0.5322 & -0.3780 & -0.0333 \\
\hline Ls（Y） & 0.3910 & 0.2820 & 0.1863 & -0.2625 \\
\hline Ls $(Z)$ & 0.4668 & -0.2429 & -0.3411 & -0.1268 \\
\hline $\mathrm{Li}(\mathrm{Y})$ & 0.3011 & 0.4930 & 0.0048 & -0.2691 \\
\hline $\mathrm{ChD}(\mathrm{Y})$ & 0.4963 & 0.1579 & 0.0694 & 0.1499 \\
\hline $\mathrm{ChD}(\mathrm{Z})$ & 0.3440 & -0.1786 & -0.3746 & -0.2089 \\
\hline eigenvalue & 2.8344 & 1.9905 & 1.4006 & 1.1884 \\
\hline contribution ratio & 0.3149 & 0.2212 & 0.1556 & 0.1320 \\
\hline accumulated contribution ratio & 0.3149 & 0.5316 & 0.6917 & 0.8283 \\
\hline
\end{tabular}

Table 4 Recovery of facial concavity by dento-maxillary prosthesis

\begin{tabular}{lcc}
\hline & $\begin{array}{c}\text { Maximum distance } \\
(\mathrm{mm})\end{array}$ & $\begin{array}{c}\text { Minimum distance } \\
(\mathrm{mm})\end{array}$ \\
\hline D-side with and without prosthesis & $9.08 \pm 4.78^{*}$ & $-1.52 \pm 0.63$ \\
N-side with and without prosthesis & $1.61 \pm 0.91$ & $-1.77 \pm 0.69$ \\
N- and D-sides with prosthesis & $2.98 \pm 2.04$ & $-2.33 \pm 1.63$ \\
\hline
\end{tabular}

mean \pm standard deviation Significant difference $*<0.05$

movements of the labrale superius (Ls) in the in the direction of $\mathrm{Z}$-axis and of the chelion on the D-side (Ch-D) in the direction of $\mathrm{Y}$-axis. In the 2nd principal component, factor loading of three variables were high: positive movements of the stomion (Sto) in the directions of $\mathrm{X}$ - and $\mathrm{Y}$-axis and positive movement of the labrale inferius $(\mathrm{Li})$ in the in the direction of $\mathrm{Y}$-axis. In the 3rd principal component, the factor loading of only one variable, positive movement of the subnasale $(\mathrm{Sn})$ in the in the direction of $\mathrm{Z}$-axis, was high. In the 4th principal component, the factor loading of two variables was high: positive movements of the alare on the D-side (Al-D) in the in the direction of $\mathrm{Z}$-axis and the stomion (Sto) in the in the direction of $\mathrm{X}$-axis. (Table 3)

\section{Recovery of facial concavity by dento-maxillary prosthesis}

The distance of the gaps between the superimposed 3-D data (maximum and minimum distances between the superimposed surfaces) and volume changes were analysed. The maximum distance between the superimposed surfaces and volume change after prosthesis placement were significantly greater on the D-side. No significant differences were noted on the $\mathrm{N}$-side between with and without prosthesis conditions or between the $\mathrm{D}$ - and $\mathrm{N}$-sides during prosthesis placement (Table 4).

\section{Discussion}

1. Usefulness of the non-contact 3-D digitiser, and objectives, and methods of this study

For aesthetic change of facial morphology after maxillectomy, reliable 3D identification of defectinduced facial disfigurement and recovery by reconstructive surgery and/or dento-maxillary prosthesis is important. $^{24-26}$ Previously, facial morphology measurement data were mainly 2-dimensional. ${ }^{27-29}$ Stoner ${ }^{30}$ was one of the first to present an image-based analysis for soft tissue diagnosis. Recent advances in 3D-CT and 3D-MR technologies have aided in achieving these objectives. However, problems, such as exposure of X-rays, dental metal artefacts, and acquisition time, remain as disadvantages. To overcome these, many reported manual methods exsist for facial soft tissue analysis. These methods quantitatively analyse facial morphology changes, (e.g., the facial impression method, ${ }^{31}$ direct measurement of the body, ${ }^{32}$ stereoscopic photography, ${ }^{33}$ and moiré topography ${ }^{34}$ ), but these are not widely employed because they are complex, inconsistent accuracy, reproducibility and injurious properties.

Recently, a new and precise analytical apparatus, the non-contact 3D digitiser, is frequently used in the fields of oral and maxillofacial surgery, plastic and reconstructive surgery, aesthetic surgery, and dentistry. ${ }^{10-14}$ The Morpheus 3D in this study scans surfaces with a semiconductor laser slit beam, and acquires images in the frontal and bilateral $45^{\circ}$ directions using a single CCD camera. These image data in the 3 directions are reconstructed into a $3 \mathrm{D}$ image. The resolution is $0.2 \mathrm{~mm}$, the acquisition time was eight seconds in each direction, and all problems with exposure of X-rays, dental metal artefacts, acquisition time, and operational issue relating to 3D-CT and/or 3DMR were solved by this novel technology.

The clinical usefulness of reproducing facial 


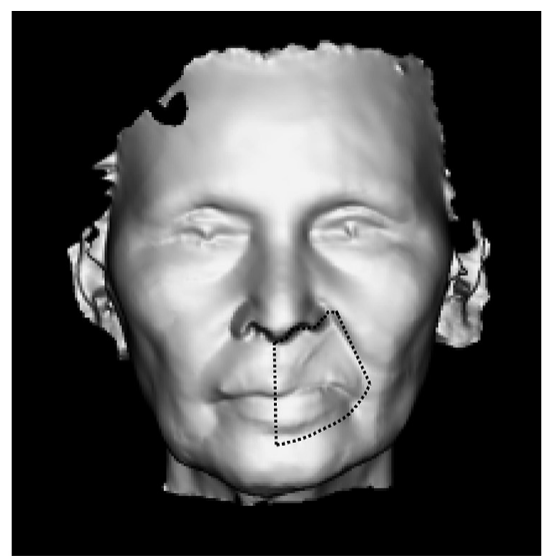

(A)

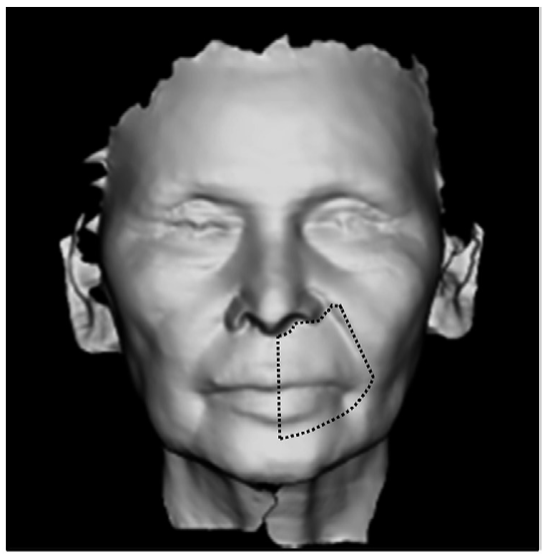

(B)

Fig. 9 Summary of recovery of facial appearance Aramany's Classification I and II facial deformities formed by partial maxillectomy (A), and summary of recovery of facial appearance with dento-maxillary prosthesis placement (B).

morphology by laser scanning has been demonstrated in many studies. ${ }^{35-39}$ The important point of laser scanning is acquisition of high-quality threedimensional facial images. Body movement-induced distortion of images was a problem with previouslaser-scanning devices, but the acquisition time of Morpheus 3D is eight seconds per direction and a total twenty-four seconds for three directions, which is very short, thereby solving this problem. However, black and brown eyelashes and eyebrows absorb the laser beam, resulting in empty spaces in the area around the eyelashes and eyebrows. This weakness in reproducing the regions around the eyelids (upper face) may be a disadvantage. To supplement this disadvantage, it is necessary to repeat scanning as needed when a defect is generated in the scanned image. We believe that with the rapid development of the laser scanning technology, a face with fantastic details will be provided and existing drawbacks will be easily resolved in the near future.

\section{Interpretation of the results of principal compo- nent analysis and recovery of facial concavity by dento-maxillary prosthesis}

The coordinate values (X, Y, Z) were measured at the reference points throughout the upper face, midface, and lower face with and without dento-maxillary prosthesis. The coordinate values at each reference point without prosthesis were subtracted from those with prosthesis, and the direction and amount of movement were analysed. Significant changes in the amount of movement were noted in nine items, and these items were subjected to principal component analysis. In the principal component analysis, when the accumulated contribution ratio of a minimum number of principal components approached 100\%, it was indicated 'favourable meaningfulness was given'. ${ }^{22,23}$ The nine measurement items were summarised into four principal components, and the accumulated contribution ratio was $83 \%$, indicating that more than $80 \%$ of all data were absorbed, based on which, the results of this analysis were jud ged as significant.

In the 1st principal component, the factor loading of two variables, positive movements of the labrale superius (Ls) in the direction of $\mathrm{Z}$-axis and chelion on the D-side (Ch-D) in the direction of $\mathrm{Y}$-axis, were high. This may be the principal component indicating prosthesis placement-induced movement of the upper lip and anterior cheek toward the anterosuperior direction. In the 2 nd principal component, the factor loading of three variables, positive movements of the stomion (Sto) in the directions of $\mathrm{X}$ - and $\mathrm{Y}$-axis and the labrale inferious ( $\mathrm{Li}$ ) in the directions of $\mathrm{Y}$-axis, were high. This may be the principal component indicating changes in the lower lip and mentolabial groove constituting of the lower face with movement of the upper lip toward the side of prosthesis placement. In the 3 rd principal component, the factor loading of one variable, positive movement of the subnasale $(\mathrm{Sn})$ in the in the directions of $\mathrm{Z}$-axis, was high, indicating that this was the principal component of forward movement of the subnasal point. In the 4th principal component, factor loading of two variables, positive movements of the alare (Al-D) on the D-side in the direction of $\mathrm{Z}$-axis and the stomion (Sto) in the direction of $\mathrm{X}$-axis were high, indicating that prosthesis placement-induced forward movement of the nasal alar and movement of the upper and lower lips toward the side of prosthesis placement were the principal components.

The principal component analysis demonstrated that facial morphology with defect of Aramany classification Class I or II mainly recovered at the upper labial, nasal alar, and subnasal points, i.e. in the midface region, and the recovery of the lower lip and mentolabial groove, i. e. in the lower face was additional. These changes were due to upper and forward movement of facial soft tissues explaining approximately $80 \%$ of overall changes. Furthermore, the 

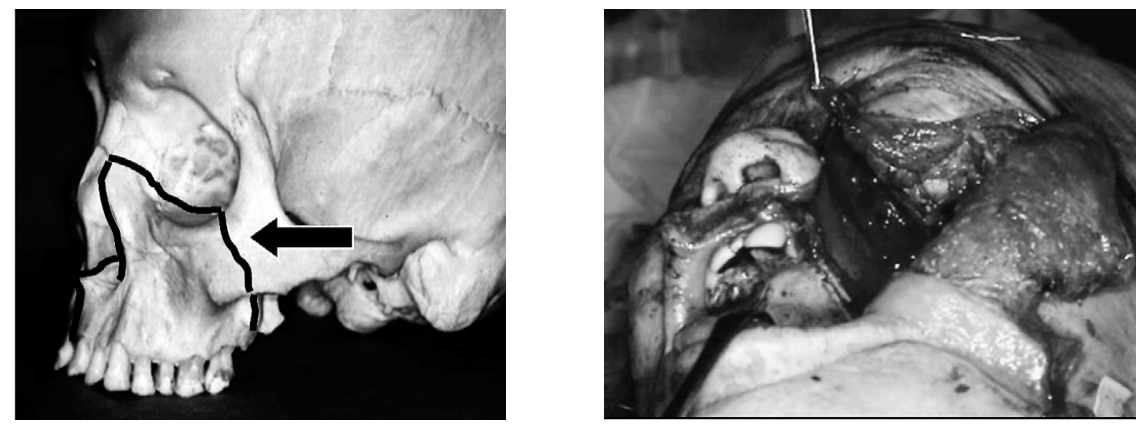

(A)
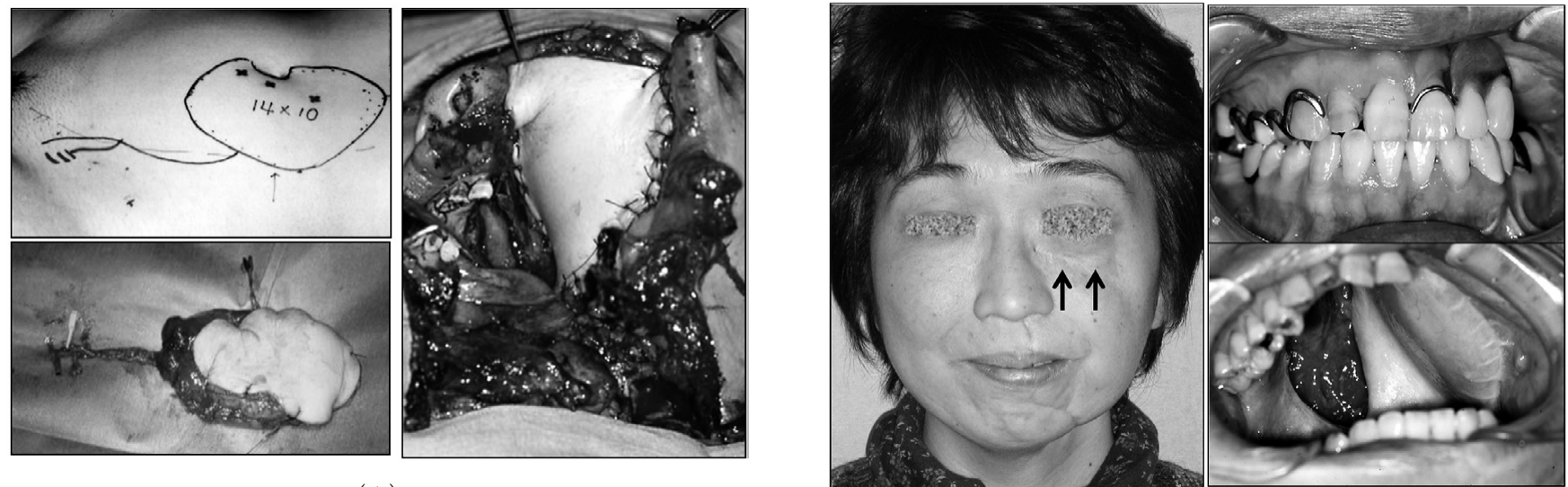

(A)

(A)

Fig. 10 Limitations of the dento-maxillary prosthesis

((A) Total maxillectomy, (B) Reconstruction with free rectus abdominis musculocutaneous flap, (C) Dento-maxillary prosthesis)

Dento-maxillary prosthesis has limited usefulness for defects resulting from total maxillectomy. Recovery of the orbital rim, i. e., concavity of the subcilliary region, with dento-maxillary prosthesis is impossible. In such cases, reconstruction with auricular or costal cartilage grafting is essential, and significant for determining an aesthetic reconstruction after total maxillectomy.

analysis of the superimposed 3-D data indicated that prosthesis placement returned the D-side contour to a state comparable with that of the $\mathrm{N}$-side, i.e., symmetrical.

Overall, it was quantitatively demonstrated that facial deformities resulting from partial maxillectomy were due to concavities from the subnasal point to the mentolabial groove in the vertical range, and from the region around the nasal alar to the anterior cheek in the lateral range. Facial features anterolaterally expanded on the D-side in volume with the prosthesis. Interestingly, facial disfigurement extended to the lower face in patients with the defects of Aramany classification Class I and II, and improvement of these deformities was also observed with the prosthesis (Fig. 9).

Using the non-contact 3D digitiser, the direction and amount of movement of the facial components within the mid- and the lower face regions were 3dimensionally determined, enabling rapid and accurate identification of facial deformities induced by the defects of Aramany Class I and II, and recovery of the facial morphology with prosthesis.

\section{Usefulness and limitations of dento-maxillary prosthesis}

This study demonstrated that dento-maxillary prosthesis placement is a promising method for aesthetic recovery of the defects of Aramany classification Class I or II; however, they have limited usefulness for defects resulting from total maxillectomy (Fig. 10 (A)). Recovery of the orbital rim, i. e., concavity of the subcilliary region, with dento-maxillary prosthesis is impossible, as shown in Fig. 10 (B) (C). In such cases, reconstruction with auricular or costal cartilage grafting is essential, and significant for determining an aesthetic reconstruction after total maxillectomy. ${ }^{1-4,7,8}$

\section{Conclusion}

Using a non-contact 3D digitiser, partial maxillectomy- and dento-maxillary prosthesis-induced facial morphology changes were objectively evaluated. This study may serve as an index for deciding on prosthesis morphology, i.e., an index used by physicians and patients to arrive at a common goal. This is the first step toward our final objective of practical use as an evaluation tool. This is the first study to analyse of prosthesis-induced changes in facial morphology 
after partial maxillectomy with a non-contact 3D digitiser.

As the facial impressions vary depending on the preferences and other factors, simply achieving lateral symmetry is not sufficient. Further studies should examine the amount of facial organisational changes that are objectively and subjectively favourable. ${ }^{1,40,41}$

\section{References}

1. Yokoo S, Kurita T. Oral and maxillofacial reconstruction. In: Kirita T, Omura K (eds). Oral Cancer -Diagnosis and Therapy-. Tokyo: Springer 2015: 231-272.

2. Granick MS, Ramasasty SS, Newton ED, et al. Reconstruction of complex maxillectomy defects with the scapular-free flap. Head Neck 1990; 12: 377-385.

3. Baj A, Youssef DA, Monteverdi R, et al. Reconstruction of partial maxillectomy defects with the double-barrel fibula free flap. Acta Otorhinolaryngol Ital 2010; 30: 299-302.

4. Lansford CD, Futran ND, Izzard ME. Reconstruction of maxilla. In: Werning JW(ed). Oral Cancer. New York: Thieme, 2007: 226-236.

5. Kosaka M, Sumita YI, Otomura T, et al. Differences of salivary cortisol levels between long-term and short-term wears of dento-maxillary prosthesis due to head and neck cancer resection. J Prosthodont Res 2014; 58: 41-47.

6. Aimaijiang Y, Otomura T, Taniguchi H. Relationships between perceived chewing ability, objective masticatory function and oral health-related quality of life in mandibulectomy or glossectomy patients with a dento-maxillary prosthesis. J Prosthodont Res 2016; 60: 92-97.

7. Martin JW, Lemon JC, Chambers MS. Oral prosthetic rehabilitation. In: Werning JW(ed). Oral Cancer. New York: Thieme, 2007: 278-287.

8. Conroy BF, Branemark PI, Blenkinsopp PT. Prosthetic restoration and rehabilitation of the orofacial cancer patient. In: Shah JP, Johnson NW, Batsakis JG (eds). Oral Cancer. London, Informa Healthcare, 2011: 401-457.

9. Hajeer MY, Millett DT, Ayoub AF, et al. Application of 3D imaging in orthodontics. part I. J Orthod 2004; 31: 62-70.

10. Taghizadeh F, Leibowitz A, Ramiretz P, et al. Combining facelift with SmartLipo 1064-nm system technique overview and retrospective analysis of 56 patients. J Cosmet Laser Ther 2016; 17: 80-85.

11. Zhang Y, Zhang L, Sun X, et al. Evaluation of lower blepharoplasty treated the SmartLipo 1064-nm system and its clinical implication: A retrospective review. J Cosmet Laser Ther 2016; 18: 376-380.

12. Santler G, Karcher H, Ruda C. Indications and limitations of three-dimensional models in craniofacial surgery. J Craniomaxillofac Surg 1998; 26: 11-16.

13. Gaggl A, Schlters G, Santler G, et al. Clinical and magnetic resonance findings in the temporomandibular joints of patients before and after orthognathic surgery. Br J Oral Maxillofac Surg 1999; 37: 41-45.

14. Ploder O, Klug C, Backfrider W, et al. 2D- and 3D-based measurements of orbital floor fractures from CT scans. J Craniomaxillofacial Surg 2002; 30: 153.

15. Aramany MA. Basic principles of obturator design for partially edentulous patients. Paer I: classification. J Prosthet Dent 1978; 40: 554-557.

16. Armary MA. Basic principles of obturator design for partially edentulous patients. Paer II: design principles. J Prosthet Dent 1978; 40: 656-662.

17. Konno A, Okamoto Y, Hanazawa S, et al. Analysis of the long-term results of our combination therapy for maxillary cancer with particular reference to treatment od T4 and N1-3 maxillary cancer, Auris Nasus Larynx 1985; 2: 147-155.

18. Sakai S, Kubo T, Mori N, et al. A study of the late effects of radiotherapy and operation on patients with maxillary cancer. Cancer 1988; 62: 2114-2117.

19. Gwilliam JR, Cunningham SJ, Huston T. Reproducibility of soft tissue landmarks on three-dimensional facial scan. Eur J Orthod 2006; 28: 408-415.

20. Powell N, Humphreus B. Proportions of the aesthetic face. New York: Thime-Stratton Inc, 1984: 1-11.

21. Naini FB. Clinical diagnostic records, natural head position a d craniofacial anthropometry. In: Facial aesthetics. Oxford: Wiley-Blackwell, 2011: 71-85.

22. Masui I, Honda T, Ishii $\mathrm{H}$, et al. An analysis of soft tissue profile changes following mndiular set back using principal component analysis. Jpn J Jaw Deform 1994; 4: 26-36.

23. Yokoo S, Komori T, Watatani S, et al. Aesthetic changes in lateral lip profile after sagittal split ramus osteotomy -Inplication for informed consent ${ }^{-}$. Asian J Oral maxillofac Surg 2002; 14: 70-77.

24. Lin SY, White GE. Mandibular position and head posture as a function of eye dominance. J Clin Pediat Dent 1996; 20: 133-140.

25. Jeffrey WB, Steven JL. Evaluation of dental midline position. Semin Oethod 1998; 4: 146-152.

26. Ferrario VF, Sforza C, Serrao G, et al. A direct in vivo measurement of the three-dimensional orientation of the occlusal plane and of the saggital discrepancy of the jaws. Clin Orthod Res 2000; 3: 15-22.

27. Harnet JC, Lombardi T, Maniere-Ezvan A, et al. Transversal craniofacial growth evaluated on children dry skulls using V2 and V3 canal openings as references. Surg Radiol Anat 2013; 35: 757-763.

28. Krimmel M, Breidt M, Bacher M, et al. Three-dimensional normal facial growth birth to the age of 7 years. Plast Reconstr Surg 2015; 136: 490e-501e.

29. Bergmann RT, Waschak J, Borzabadi-Farahani A, et al. Longitudinal study of cephalometric soft tissue profile traits between the age of 6 and 18 years. Angle Orthod 2014; 84: 48-55.

30. Stoner MM. A photometric analysis of the facial profile: a method of assessing facial change induced by orthodontic treatment. Am J Orthod 1955; 41: 453-469.

31. Tanaka Y, Gold H, Pruzansky S. New facial impression technique in maxillofacial. Maxillofac Prosthet 1979; 2: 11-14.

32. Farkas LG. Anthropometry of the attractive North American Caucasian face. In: Head and face, $2^{\text {nd }}$ ed. Raven Press 1994, 159-183.

33. Ras F, Habets L, van Ginkel FC, et al. Quantification of facial morphology using stereophotogrametrydemonstration of a new concept. J Dent 1996; 24: 369-374.

34. Kawai T, Natsume N, Shibata H, et al. Three-dimensional analysis of facial morphology usung moiré stripes Part II. Analysis of normal adult. Int J Oral Maxillofac Surg 1990; 19: 359-362.

35. McCance AM, Moss JP, Fright WR, et al. A threedimensional analysis of soft and hard tissue changes following bimaxillary surgery. Br J Oral Maxillofac Surg 1992; 30: 221-232.

36. McCance AM, Moss JP, Fright WR, et al. A threedimensional analysis of 16 skeletal class III patients following bimaxillary surgery. Br J Oral Maxillofac Surg 1992; 30: 221-232.

37. Morris DO, Illing HM, Lee RT. A prospective evaluation of Bass, Bionator and Twin Block appliance. Part II-The soft tissues. Eur J Orthod 1998; 20: 663-684. 
38. Shamique FH, Moss JP, Hennessy R. Three-dimensional assessment of the effects of extraction and nonextraction orthodontic treatment on the face. Am J Orthod Dentofacial Orthod 2002; 121: 244-256.

39. Sforza C, Elamin F, Rosati R, et al. Three-dimensional assessment of nose and lip morphology in North Sudanese subjects with Down syndrome. Angle Orthod 2011; 81: 107-114
40. Yokoo S, Makiguchi T. Mandibular reconstruction -vascularized bone and regenerative bone marrow grafts ${ }^{-}$Int $\mathbf{J}$ Microdento 2013; 4: 66-72.

41. Yokoo S, Sarukawa S. What is the standard treatment for mandibular reconstruction? -Sharing the objective of treatment and awareness of issues ${ }^{-}$. J Jpn Oral Oncol 2015; 3: 21-29. 